\title{
ZU ANGLIA XII, 375 ff.
}

Auf Sarrazin's aufsatz nochmals eingehend zu antworten halte ich für unnötig.

Wenn jemand, wie es S. tut, annimmt, dass die Fat. Ap. dichterisch der Elene näher ständen als der Juliana, so kann man sich auch nicht wundern, wenn er zeitlich Fat. Ap. gerade vor das vollendetste werk Cynewulf's, vor die Elene, setzt. Auch von seiner ansicht, dass Fat. Ap. den schluss zum Andreas gebildet hätten, obgleich gerade die geschichte des Andreas ganz anders in den Fat. Ap. erzählt wird, lässt sich S. nicht abbringen, er wird aber auch kaum jemand von der richtigkeit seiner meinung tberzengen. Wenn ferner $S$. die 'widersprttche' zwischen der Andreaslegende in Fat. Ap. mit der darstellung im Andreas nur 'abweichungen' nennen will, so ist dies geschmackssache. Die tatsache bleibt bestehen, dass Fat. Ap., die nach S. den schluss des Andr. bildeten, die im Andr. enthaltene legende gar nicht kennen! Ein vergleich der reihenfolge der apostel in Fat. Ap. ergibt, dass Cynewulf weder den evangelisten Matthäus und Lukas, noch den anordnungen Beda's, des Venantius oder des Breviariums folgt, sondern sich allen gegenuber umstellungen erlaubt. Warum also hätte er nicht den Matthäus auf den Andreas können folgen lassen? Eine vergleichung der Fata, soweit sie den Andreas betreffen, mit dem s. 381 abgedruckten texte Beda's zeigt, wie wenig ubereinstimmendes beide haben. S. selbst sagt: 'Beda's notizen sind meist ausfuhrlicher, aber bisweilen geben die Fata genaueres, so bei Philippus und besonders bei 'Thomas'. Die schilderung von dem leben und tode des Matthäus widerspricht geradezu der Beda's (und somit auch wol der des Breviariums). Man sieht also, dass Sarrazin's behauptung: 'Der inhalt unseres gedichtes stimmt im wesentlichen uberein mit dem, was das Breviarium erzählt; ebenso auch mit den angaben von Beda's martyrologium' falsch ist.

Dies möge genugen. Auch bei meiner ansicht, dass sich S. direkt widersprochen habe (vgl. s. 375 anm.) bleibe ich.

GOHLIS-LEIPZIG.

R. WÜLKER. 